\title{
Criminologie
}

\section{Le citoyen et la justice}

\section{Louis Huot et Jocelyn Giroux}

Volume 12, numéro 1, 1979

Justice et public

URI : https://id.erudit.org/iderudit/017098ar

DOI : https://doi.org/10.7202/017098ar

Aller au sommaire du numéro

Éditeur(s)

Les Presses de l'Université de Montréal

ISSN

0316-0041 (imprimé)

1492-1367 (numérique)

Découvrir la revue

Citer cet article

Huot, L. \& Giroux, J. (1979). Le citoyen et la justice. Criminologie, 12(1), 43-56. https://doi.org/10.7202/017098ar d'utilisation que vous pouvez consulter en ligne.

https://apropos.erudit.org/fr/usagers/politique-dutilisation/ 
LE CITOYEN ET LA JUSTICE

Louis Huot Jocelyn Giroux

L'objectif de la recherche, dont nous rapportons ici les résultats ${ }^{1}$, visait à identifier les mécanismes du système pénal et à voir comment, dans l'administration quotidienne, ceux-ci interviennent et s'actualisent. Ce faisant, on s'aperçoit que l'image idéale de la justice est loin de correspondre à la réalité. Lorsqu'une victime d'une agression appelle la police, elle déclenche - souvent sans le savoir - la mise en marche d'une machinerie lourde aux rouages multiples et complexes, qui se meurt lentement et dont l'efficacité laisse perplexe. L'analyse de cette longue partie d'échecs, qui a pour enjeu la libération ou la condamnation d'un suspect, s'avère indispensable à l'étude des attitudes du public envers la criminalité et la politique criminelle.

Pour ce faire, nous avons, pendant plus de cinq mois, à raison de deux à quatre jours par semaine, fréquenté les tribunaux, arpenté les salles des pas perdus, discuté avec des policiers, des journalistes, des témoins et des enquêteurs. Constamment aux aguets, nous avons observé, écouté, regardé, noté faits et gestes ; ce qui nous a permis de nous familiariser avec le monde hermétique de la justice. Nous nous sommes initiés peu à peu au langage spécialisé des experts (avocats, procureurs, juges), aux pratiques en vigueur, aux astuces et aux procédures judiciaires, à la prose rigoureuse des articles de loi. Nous avons pu de cette façon saisir la signification et la portée des diverses étapes du procès, le sens des jeux de coulisse et des apartés entre les différents acteurs.

En plus de cette activité de spectateurs assidus, nous avons interviewé plusieurs petits commerçants qui avaient fait l'objet de vol qualifié.

C'est le fruit de ces observations et de ces entrevues qui est résumé dans le rapport inédit sur "Les petits commerçants victimes de vol à main armée ».

1. Voir le rapport de recherche inédit, \&e citoyen victime de vol qualifié : sa place dans le processus judiciaire $\gg$, dans le cadre des études sur les réactions de l'opinion publique (Groupe de recherche sur les attitudes envers la criminalité (G.R.A.C.) du Centre international de criminologie comparée C.I.C.C.), vol. 6, 337 pages, Montréal, 1977. 
Quant à nous, notre rapport s'est divisé en deux parties : l'une « illustrative »; l'autre, analytique. Dans le livre I : « Anatomie d'un vol qualifié » nous avons décrit sous la forme d'un scénario le vol d'une bijouterie par trois délinquants. Les ressemblances avec des faits et des personnages réels ne sont ni fictives, ni involontaires.

Toutes les situations décrites et tous les propos relatés sont véridiques.

Pour examiner tous les aspects du fonctionnement de la justice criminelle, nous avons greffé sur l'événement principal, un vol de $\$ 80000$ de bijoux, des incidents ou des situations qui appartiennent à d'autres infractions similaires ou à d'autres protagonistes, de telle sorte qu'on y retrouve une condensation d'événements ; ce qui amplifie l'importance de certains comportements et de certaines façons d'agir des parties en présence tout au long des étapes qui vont de la commission d'un délit à la condamnation des coupables.

Le livre II constitue une analyse plus détaillée des faits décrits en première partie. C'est dans celui-ci que nous voyons plus en profondeur le sort réservé à la victime par le système de justice criminelle. Décortiquant le processus judiciaire criminel à partir de la préenquête jusqu'au prononcé de la sentence, dans ses moindres détails, nous analysons au fur et à mesure les interactions entre les agents et mécanismes du système avec les victimes d'un délit. Aucune étape de la procédure, ou presque, du système judiciaire n'a été oubliée et chacune a été analysée par rapport aux répercussions qu'elle peut avoir vis-à-vis de la victime aux prises avec ledit système.

Dans ce bref article, nous tenterons donc de décrire sommairement les conclusions et remarques qui ont été tirées de la recherche et nous ferons part des commentaires suscités par l'analyse des faits observés au cours du processus judiciaire.

La justice rendue par nos tribunaux ne serait-elle qu'un mythe ? Comment pourrions-nous expliquer le désenchantement qui suit les premiers contacts du citoyen avec la cour? Le système de justice criminelle ne serait-il construit qu'en fonction de l'accusé ? Les agents du système (avocats, juges, criminologues et policiers) ne seraient-ils que des agents de l'accusé ? Quelle place réserve-t-on à la victime dans notre système de justice criminelle? 
Voilà quelques questions que nous pourrions soulever à titre d'entrée en la matière ; elles résument assez bien le fond du problème que nous avons tenté d'analyser.

«Il n'y a pas de justice », \& il y a deux justices », « la justice, ce n'est pas pour nous », voilà des commentaires que nous avons souvent entendus de la bouche de citoyens frustrés par le système. Pour ceux-ci la justice ne sert à rien, ce n'est que du théâtre, c'est une farce : mieux vaut éviter d'avoir recours aux tribunaux.

Suite à ces commentaires plus ou moins flatteurs à l'endroit d'une institution aussi importante, il convenait certes de s'interroger sur les finalités du système de justice criminelle. Quels sont ses intérêts? Ceux de l'État qui y voit un moyen de répression, diront certains, ou ceux d'un Etat qui cherche à endormir ses sujets dans une illusion d'ordre social ? Il se pourrait bien aussi que ce soit tout simplement les intérêts des classes professionnelles qui y gagnent très bien leur vie (juges, avocats, agents de probation, agents de libération conditionnelle, etc.).

Nous verrons dans le condensé qui va suivre comment le système judiciaire actuel ne dessert pas, ou mal, les citoyens qu'il touche et cela à travers trois thèmes principaux : les lenteurs judiciaires, l'absence de participation du citoyen et le caractère antisocial des tribunaux.

\section{Les lenteurs judiciaires}

Envisagé comme une forme de communication entre le système et le public en général et plus particulièrement les délinquants en puissance, le «syndrome du délai » communique à ceux-ci le message que le crime et ses auteurs ne constituent pas vraiment une matière urgente d'intérêt public ${ }^{2}$.

Ce phénomène curieux d'abus des procédures judiciaires est ressorti tout au long de nos observations au Palais de justice. Ce processus dilatoire a déjà fait l'objet de critiques sérieuses de la part des professionnels de la justice ${ }^{\mathrm{s}}$.

2. Penegan, Appraising the System of Criminal Law, Its Processes and Administration, 47 N.C.L., Rev. 69, 150 (1968).

3. Commission d'enquête sur l'administration de la justice en matière criminelle et pénale au Québec (La justice criminelle, vol. 1-2-3-4), 1969. 
Dans un article publié dans la Presse ${ }^{4}$, le juge Albert Dumontier, juge en chef de la Cour des sessions, à Québec, dénonçait les abus envers l'aide juridique. Selon lui, les avocats abusent des procédures depuis la création de l'aide juridique. Il en donne comme exemple le fait que peu d'accusés plaident coupable lors de leur comparution même quand il s'agit de cas de flagrant délit, que la plupart choisissent, dans un premier temps, un procès devant juge et jury pour opter, quand vient le temps de fixer une date, pour un procès devant juge seul et ensuite plaider coupable dans les trois quarts des cas. Ce qui amène, dit-il, une surcharge des rôles et des retards considérables.

Il n'en tient toutefois pas responsables les seuls avocats de pratique privée, bénéficiaires de mandats de l'aide juridique. Au contraire, affirme-t-il, les permanents de l'aide juridique jouent le même jeu.

Toutefois, alors que les avocats de la pratique privée le font «pour accumuler des honoraires », ceux de l'aide juridique, selon le juge Dumontier, se servent de ces moyens pour «donner de l'importance à leur fonction et bénéficier de la dynamique du tempo s.

Il explique qu'en effet les prévenus ont avantage à plaider coupable plusieurs mois, sinon plusieurs années, après la commission de leur crime, alors que l'opinion publique n'est plus surchauffée, car le juge se montrera souvent plus clément.

Le rapport de la CECO, publié en septembre 1976, décrit très bien ce processus, à l'origine des sentences judiciaires ${ }^{5}$. Il y a tout d'abord la remise qui consiste dans le fait de reporter l'audition d'une cause à une date ultérieure. "Personne dans le système judiciaire n'ignore que la remise est pour la défense un outil privilégié qui est utilisé quotidiennement comme un moyen de parvenir à un acquittement. La remise apporte, en effet, du délai et qui dit délai, dit diminution du souvenir des témoins, pertes de mémoire, affaiblissement du récit, démotivation et usure des témoins qui doivent inlassablement se présenter à la cour, diminution de l'intérêt des policiers, du procureur et du juge, perte d'impact d'une preuve étalée par morceaux sur une longue période, etc. »

4. La Presse, 9 février 1977 (Juge Albert Dumontier).

5. Rapport de la C.E.C.O., 1976, La lutte au crime organisé au Québec, rapport d'enquête sur le crime organisé et recommandations. 
La remise peut être demandée pour diverses raisons :

Défense : «M. le juge, j'aimerais faire une remise, pour contre-interroger la victime, $\mathrm{j}$ 'aurais besoin des notes sténographiques et il faudrait une remise pour les avoir et prendre le temps de les lire soigneusement...

Parfois, c'est la Couronne qui est prise en défaut, n'ayant pas assigné ses témoins ou écrit les subpœena.

Les moyens employés par la défense pour obtenir les remises sont variés : l'avocat acceptera plusieurs mandats dans la même journée, l'accusé répudiera son avocat le matin de l'audition, un témoin de la défense sera absent ou l'on invoquera un manque de temps pour prendre connaissance du dossier et des témoignages des précédents. Le «marchandage » des juges a aussi pour effet de retarder les procédures.

Il en va de même pour les continuations de causes. Certains avocats de la défense s'arrangeraient pour que le nombre de jours retenus pour l'audition de la cause soit insuffisant, en sousévaluant volontairement la durée de la cause auprès du maître des rôles, ou bien en prolongeant indûment leur contre-interrogatoire, ou encore en étirant le temps au moyen de requêtes orales fondées. On peut aussi interrompre l'instance pendant une demijournée ou une journée complète sous le prétexte qu'on est retenu dans une autre cause, ou en réclamant un ajournement pour obtenir la transcription du témoignage d'un témoin, ou pour préparer la défense de l'accusé, comme si l'année écoulée depuis la mise en accusation n'avait pas été suffisante pour le faire.

Une étude réalisée en 1970 sur le fonctionnement des cours criminelles de Chicago par deux chercheurs américains, Baufield et Anderson ${ }^{6}$, a révélé que les chances de réussite des poursuites décroissent généralement avec la longueur du délai entre l'arrestation et la finalisation de la cause, le nombre de présences de l'accusé à la cour et le nombre de présences des témoins en cour.

Sur ce dernier point, les chercheurs ont constaté que l' usure $>$ des témoins est une pratique utilisée couramment et généralement avec succès. Une relation directe a pu être établie entre

6. Baufield et Anderson, Continuances in the Cook County Criminal Courts \, 35, University of Chicago Law Review 259, p. 287-288 (1968). 
la durée des causes et le taux des condamnations. Ainsi, sur l'échantillon global, on a pu constater que le pourcentage de condamnations qui était de $92 \%$ pour les causes requérant d'une à quatre visites de témoins à la cour passait à $48 \%$ dans les causes requérant dix-sept visites ou plus ${ }^{7}$. Peu d'observateurs du milieu pourraient contester le fait que ces conclusions américaines ne puissent tout aussi bien s'appliquer au Canada ou au Québec.

Évidemment, les remises demandées par la Couronne diffèrent dans leur sens de celles de la défense.

Mais peu importe en fait qui demande la remise car, quel que soit le cas, on joue consciemment ou inconsciemment sur une continuation prolongée des causes. L'effet déplorable créé chez les témoins ou victimes demeure le même. Quant à l'inculpé, il paie souvent les délais par des mois de détention avant procès et ce, dans des conditions particulièrement pénibles.

L'influence des remises et des continuations de causes ne se fait pas sentir seulement sur le pourcentage des condamnations par le biais des témoignages affaiblis ou démotivés des victimes. Le facteur économique est loin d'être négligeable; à chaque remise, même si le nontant est dérisoire, les victimes ou témoins sont dédommagés ; les juges et procureurs de la Couronne dépensent un temps énorme sur une même cause pendant qu'ils pourraient en régler une multitude d'autres.

Comme le disait un juge des Sessions lors d'une affaire : ه Pendant que vous faites venir ici deux policiers qui perdent un avant-midi à cause d'une mauvaise organisation, il y a des crimes, des hold-up qui se commettent à l'extérieur de la cour. \$ On peut ajouter à ces dires le salaire des policiers mobilisés en cour à ne rien faire, leurs heures payées à attendre pour se voir fixer une autre date à laquelle ils devront revenir...

Une des conséquences extrêmement importante de cette lenteur qui détruit l'image de notre système de justice, c'est la perception qu'en ont les témoins ou victimes qui sont les sujets les plus extérieurs à cette administration tout en étant, ne l'oublions pas, les personnes clés de ces procès.

7. Etude de Institute for Law and Social Research, Washington (D.C.), « A Study of Witness Cooperation with district of Columbia Prosecutor », Frank J. Camavale, Jn., p. XL, 1975. 
Tous les professionnels de l'administration de la justice pénale admettent sans ambages que sans la collaboration des citoyens, ni l'accroissement du personnel, ni l'amélioration du personnel, ni l'augmentation des budgets ne permettraient aux forces de l'ordre de contenir adéquatement le phénomène de la criminalité ${ }^{8}$.

Pourtant, si le témoin ou la victime qui constate le crime, qui le rapporte à la police et qui en témoigne par la suite devant la cour est un personnage capital du système, c'est aussi le plus méconnu et le plus maltraité. Au-delà d'une quarantaine d'entrevues avec des victimes de hold-up qui avaient vécu des expériences à la cour nous ont démontré l'insatisfaction prononcée de ces citoyens désireux de témoigner. La liste des inconvénients qui échoient aux témoins ou victimes est considérable : perte de temps, perte monétaire, mauvaises conditions matérielles dans les palais de justice, traitement cavalier dont ils font l'objet de la part des avocats, des juges, etc. Mais laissons parler des victimes de cette expérience. Voici trois témoignages de différents citoyens :

Arrangez-vous avec vos problèmes moi je suis fatigué, et je me suis fâché.

J'ai eu des ennuis. Je suis allé en cour quatre ou cinq fois pour cette affaire.

C'est une perte de temps. On perd deux à trois jours de travail. Le mot d'ordre est donné et tout le monde le sait. N'importe quel commerçant évite de porter plainte à cause de cela. C'est une perte de temps qui ne donne rien. Je pense que c'est un fait : on ne peut vraiment pas dire qu'on est protégé.

En fait, les victimes lors des entrevues ont montré une sévère démotivation à l'égard du système. Les remises, la perte de temps fastidieuse ajoutent encore à leur victimisation. La lenteur judiciaire amène la victime à penser qu'elle n'est que le jouet du système, que de toute façon ça ne donnera rien.

A la cour, il y a beaucoup de pertes de temps. La perte de temps entre les juges, les avocats et les remises. J'ai déjà vu cinq à six remises pour la même cause... parce qu'un avocat est occupé dans une autre cause, et n'a pas le temps de venir à celle-là ou parce qu'il y a défaut. Parce qu'il y a un témoin qui n'est pas là ou que... en regardant tout cela... on reste avec un goût amer...

8. U.S. National Advisory Commission. 
La rétribution des témoins est tout à fait ridicule. On peut comprendre la réticence de plusieurs victimes qui, pour un vol qualifié de $\$ 100$, vont perdre parfois quelques centaines de dollars à force de se présenter à la cour sur une période de quelques années. Comme le disait un juge de la Cour des sessions de la paix à Montréal, à qui la défense venait de demander une remise :

Les témoins qui retournent chez eux sans avoir rien à dire sont frustrés. La plupart des témoins gagnent plus en une heure que pendant une journée à la cour. Même si le fait de témoigner constitue un devoir civique, on comprendra facilement qu'en plus de tous les autres inconvénients, la perte, à toutes fins pratiques, du salaire d'une ou de plusieurs journées ne facilite pas la tâche déjà ardue de ceux qui ont à convaincre des témoins, récalcitrants au départ, de bien vouloir collaborer avec la justice.

Des rencontres avec des policiers et des enquêteurs nous ont permis de savoir qu'il arrive fréquemment que des témoins, clients d'un magasin où se produit un vol qualifié, refusent tout simplement de collaborer ou déguerpissent aussi rapidement que le voleur de peur \& d'avoir du trouble \$.

Au niveau qualitatif, les entrevues réalisées démontraient combien générales étaient l'insatisfaction et la déception des victimes.

$\mathrm{Au}$ fond, ces doléances ne représentent-elles pas un désenchantement fondé et justifié par les faits ? Il est tout de même malheureux à ce sujet que, comme nous l'a affirmé un de nos interlocuteurs :

Certains, ignorants du fonctionnement du processus judiciaire, deviennent fermement convaincus qu'une conspiration existe dans leur cause spécifique pour obstruer le cours de la justice - conspiration dont font partie le juge présidant la cause, le procureur de la poursuite et le policier-enquêteur.

Bien que seule la méconnaissance des faits procéduraux puisse prêter à une interprétation, faut-il blâmer le citoyen-victime de penser de la sorte?

\section{L'absence de participation du citoyen}

Victime ou accusé, le citoyen est un incompétent qui n'a pas voix au chapitre. 
D'après les propos des victimes que nous avons interrogées, nous avons pu constater que les plus expérimentées d'entre elles, quant à la fréquentation du Palais de justice, saisissent probablement l'essentiel de ce qui s'y déroule... Selon elles, il s'agit d'un jeu dont l'avocat de la défense est la vedette. Son rôle serait celui de confondre les témoins et d'innocenter son client ; et cela par n'importe quel moyen. Plus les honoraires seront élevés, plus son jeu sera habile et vice versa. Quant au procureur de la Couronne, il se contente de donner la réplique. Le juge, pour sa part, prendra une décision, mais sans avoir, auparavant, indiqué de quel côté il était, par son attitude ou ses interventions. Et en général, dit-on, c'est du bavardage, du \& niaisage $\$ .$.

Il arrive malheureusement que les avocats abusent de leurs clients absolument ignorants des lois et du système de justice. Ainsi un avocat, tout aussi connu que de réputation douteuse, avait demandé à la mère d'un de ses clients une somme de $\$ 500$ pour l'octroi de la libération conditionnelle de celui-ci. Or, l'avocat qui n'a qu'un pouvoir de représentation avait menti délibérément au client qui, lui, était convaincu de sa compétence quant à l'octroi de la libération conditionnelle. Ajoutons qu'un tel octroi était facilement prévisible d'ailleurs, vu le dossier exemplaire du délinquant en question.

De la même façon, un criminaliste réputé a demandé l'option « juge et jury » pour un client accusé d'un vol de $\$ 5,95$. Par la suite, un interlocuteur averti nous racontait à ce propos :

Imagine-toi la galette que ça va coûter au gars pour un vol de $\$ 5,95$. Il va probablement lui dire après, qu'il a fait des efforts surhumains pour faire baisser l'acte d'accusation à la déclaration sommaire de culpabilité et son client qui n'y connaît rien à tout cela va se faire jouer au bout...

Et même si souvent il n'y a aucune intention d'abus, il n'en reste pas moins que dans la plupart des cas l'accusé fait les frais des tactiques de son procureur. Il n'a pas un mot à dire, ou si peu, sur la façon dont il sera défendu. Il arrive qu'un accusé soit aux prises avec des procédures criminelles pendant des années sans jamais savoir au juste ce qui se passe. Souvent l'accusé regrette de ne pas avoir plaidé coupable dès le début et ce malgré qu'il soit véritablement innocent.

La victime est encore plus passive et impuissante devant le tribunal. Elle n'est invitée à se présenter que si son témoignage 
est nécessaire à la preuve de la poursuite. Son rôle est celui d'un accessoire de la justice. Notre système judiciaire considère la victime comme une personne qui doit aider le ministère à faire condamner l'agresseur. C'est en partie le témoignage de la victime qui permettra au ministère public de présenter toute la preuve nécessaire pour la condamnation de l'accusé. Son rôle primordial dans l'élaboration de la preuve, nécessaire pour que justice soit équitable, peut être considéré comme le maillon principal de notre système judiciaire.

La victime n'est pas considérée comme une personne qui a été agressée mais plutôt comme quelqu'un qui doit aider le ministère public à faire condamner un agresseur parmi tant d'autres. Pourtant plusieurs spécialistes du système affirment sans ambages que la victime-témoin ne reçoit pas le traitement qu'elle serait en droit d'avoir. Les enquêteurs de la CECO sont de ceux-là. Plusieurs fonctionnaires du système judiciaire nous en ont également fait la remarque et si plusieurs s'accordent à dire que la victime est la grande oubliée du système, certains sont plus virulents encore dans leur dénonciation. Citons, à ce propos, les paroles d'un chroniqueur judiciaire, vieux routier du Palais de justice :

La victime est traitée comme du poisson pourri. Quand je pense à la caissière d'une banque qui s'est fait masturber par un mineur sous la menace d'un revolver! Cette petite femme-là, de vingt ans à peu près, en a pissé dans ses culottes à la comparution et elle tremblait comme une feuille lors de la reprise du procès, alors que ça fait déjà trois ans que ça a été commis. Elle est finie pour la vie, cette pauvre jeune femme. Elle travaillait simplement pour une banque et voilà qu'elle se retrouve désorganisée, qu'elle n'est pas remise et qu'elle ne s'en remettra peut-être jamais.

Et un peu plus loin, le chroniqueur judiciaire ajoute :

Promenez-vous dans les couloirs et vous allez entendre des témoins se faire insulter, se faire menacer. Il y a des citoyens qui n'ont pas assez d'être témoin ou victime qu'ils se font écœurer par des bandits, qu'ils se font appeler chez eux le soir et se font dire par exemple : mon tabarnak, demain, je te conseille de fermer ta gueule si tu veux pas te la faire casser.

Et que penser de ces propos d'une pauvre mère de famille qui s'écriait en cour lorsque mise en présence de l'agresseur de sa fille : « You killed my daughter \$. 
Certes, les paroles de notre chroniqueur judiciaire ne souffriraient pas de quelques bémols, mais n'en reste pas moins un dard de vérité qui ne doit pas être tenu sous silence.

\section{Le caractère antisocial du système judiciaire}

Ce qui ressort le plus de notre recherche c'est sans contredit l'absence flagrante de participation du citoyen au système de justice criminelle. S'il est accusé, il doit se résoudre à jouer un rôle passif et à remettre son sort entre les mains des experts qui, eux, sont seuls à connaître les ficelles de cet univers mystérieux et ésotérique. S'il est victime, sa déception sera d'autant plus grande : on le vole, l'assaille, le viole, le fraude et le système ne lui réserve aucune compensation. Au contraire, après avoir été proprement volé ou violé, il entre dans une nouvelle phase de frustrations. Le système judiciaire s'en servira pour soi-disant faire justice, venger la société et mettre hors d'état de nuire un accusé. Malheureusement, plus souvent qu'autrement le citoyen victime n'aura même pas la satisfaction de voir l'accusé mis hors d'état de nuire, soit parce que ce dernier aura eu un \& bon avocat \$ qui tire bien les ficelles, ou que le système, faute de meilleures solutions, ait cru bon de le mettre en probation ou de lui donner simplement une sentence suspendue. Quel réconfort, en effet, pour le citoyen de voir, deux semaines après avoir été volé et maltraité, l'auteur de tous ses malheurs en liberté jouissant d'une sentence suspendue.

Ce citoyen sort d'une phase épuisante qu'est celle du processus judiciaire. Pendant ces heures difficiles pour lui, à tout moment, il s'est senti exclus et oublié par le système. Personne ne s'est préoccupé de lui, si ce n'est que pour s'en servir. Il n'a rien ou à peu près rien compris de tout ce qu'il vient de vivre. Et comment pourrait-il en être autrement ? Le langage est hautement technique et plein de sous-entendus ou d'allusions, le tout entremêlé de mots d'esprit d'un jargon juridique, d'envolées oratoires. Le citoyen est tellement perdu que dans sa perplexité il en viendra quelquefois à croire que tout le scénario n'a pour but que de se payer sa tête.

Les négociations de plaidoyers (plea bargaining), qui révèlent la complicité de l'avocat de la défense et du procureur de la Couronne afin de hâter le dénouement du procès en atténuant la gravité du délit, sont incomprises des victimes qui, là aussi, 
y voient souvent un complot contre elles. On les considère comme des transactions malhonnêtes. Quant aux accusés, eux, souvent avec un sourire en coin, ils y trouvent une bonne occasion de s'en tirer à bon compte en « acceptant s un plaidoyer de culpabilité pour une faute amoindrie. La victime vit fréquemment cette procédure comme une insulte. Elle ne s'explique pas qu'un accusé puisse s'en tirer si facilement. En fonctionnant de la sorte et en engendrant autant de frustrations et d'injustices, le système judiciaire nous est apparu, à certains égards, comme comportant un caractère antisocial.

Nous en sommes arrivés à ces conclusions pour deux principales raisons. La première, c'est qu'il décourage amèrement certaines victimes d'actes criminels d'accomplir leurs devoirs de citoyens en portant plainte. Ils ne croient plus au système judiciaire et décident donc de l'abandonner. On en est arrivé à la constatation que plus un citoyen a de contacts avec les agences pénales, plus sa confiance dans les institutions s'en trouve érodée et minée. Bon nombre de commerçants victimes en viendront à adopter une attitude défaitiste et décideront de ne plus faire appel au système pénal dans l'avenir, celui-ci ne leur ayant apporté que déceptions et brimades. Certaines victimes, en plus d'avoir été complètement détroussées de tout ce qu'elles ont réussi à amasser de peine et de misère, en plus d'avoir été molestées par leur agresseur, ont dû faire de multiples démarches, bien souvent infructueuses, répondre aux questions de la police, à celles des enquêteurs de la compagnie d'assurance et à celles des avocats qui ne se gênent pas, parfois, pour les malmener. Plusieurs ont même dû vendre leur commerce et, ainsi, perdre leur gagne-pain. D'autres restent traumatisées et se procurent des moyens (chiens, matraques, armes) pour pouvoir se défendre elles-mêmes. On assiste à une escalade de la violence engendrée par l'acte du délinquant mais aussi par les imperfections du système judiciaire, ce dernier laissant maintes fois pour compte une victime qui aurait tant besoin de justice.

Mais ce n'est pas toujours ce que le système lui offre. En effet, plusieurs victimes appelées à témoigner à la barre se sentent considérées comme des accusés. Certaines, plus déçues encore, aimeraient quelques fois être traitées au moins aussi « bien » que les inculpés. Dans ces cas-là, au traumatisme causé par l'attaque violente s'ajoute celui du tribunal. L'expérience de la cour amène 
donc d'autres embêtements à des personnes déjà gravement lésées. Elles se voient négligées par les juges et avocats qui se concentrent sur le sort du \& malheureux » délinquant. Pourtant, les victimes devraient s'attendre à plus de considération et à un dédommagement quelconque de la part du système pénal.

La deuxième raison du caractère antisocial de la justice concerne l'auteur d'une infraction. Devant le marchandage dont il est l'objet, il apprend comment * déjouer > la justice. L'accusé réalise que ce qui est puni, ce n'est pas un acte qui a causé un tort à des individus ou à la société, mais c'est l'échec dans les négociations, la malchance d'avoir eu un juge sévère, l'incapacité de payer un bon avocat. En un sens, le système pénal n'offre pas l'attrait d'un exemple de la justice de cette Thémis idéale, impartiale, presque supra-humaine.

\section{Conclusion}

Certes nos remarques et conclusions sont à certains égards pessimistes. Certains y verront, à tort, de l'exagération de notre part, ce qui est le plus important, pour nous, toutefois, c'est qu'elles portent et qu'elles fassent réfléchir. Nous croyons que des réformes s'imposent, et qu'elles tardent à venir. C'est peut-être pour cette raison d'ailleurs que nous paraissons défaitistes et pessimistes. Si nous avons insisté sur certaines lacunes un peu lourdement, si, quelquefois, nos propos sont très sévères, nous nous en excusons auprès de ces puristes de la science criminologique qui savent toujours garder une objectivité à toute épreuve. Toutefois, nous désirons ardemment que ces mêmes puristes et tous ceux qui liront cet article tentent d'oublier en dernier ressort les lacunes, pour mieux réfléchir sur certains problèmes que nous avons soulevés. Parmi ceux-ci vient en premier lieu le statut qu'il conviendrait de donner à la victime.

En ce qui nous concerne, après nos multiples observations, nous arrivons à la conclusion qu'il est temps que soit donné, par le système de la justice criminelle, un nouveau statut à la victime. Ce statut consisterait tout au moins à traiter avec respect et dignité un citoyen victime d'un acte criminel, ce qui impliquerait que l'on songe, non seulement à punir le coupable, mais aussi à appliquer la loi d'indemnisation de la personne lésée. Tout comme l'accusé, la victime a ses droits et il serait temps que tous ceux qui s'occupent d'administrer notre justice criminelle (juges, politiciens, 
avocats, criminologues, etc.) lui accordent plus d'importance! Le système pénal fonctionne mal, on en conviendra, et c'est souvent grâce au courage de certaines victimes et de certains témoins que la « justice peut être rendue » et que ses rites et ses pompes peuvent demeurer crédibles. 